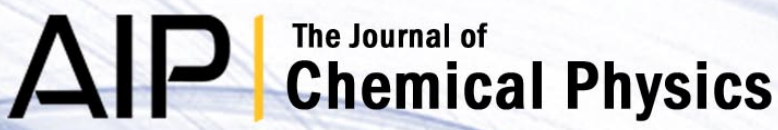

Elasticity of flexible and semiflexible polymers with extensible bonds in the Gibbs and Helmholtz ensembles

Fabio Manca, Stefano Giordano, Pier Luca Palla, Rinaldo Zucca, Fabrizio Cleri et al.

Citation: J. Chem. Phys. 136, 154906 (2012); doi: 10.1063/1.4704607

View online: http://dx.doi.org/10.1063/1.4704607

View Table of Contents: http://jcp.aip.org/resource/1/JCPSA6/v136/i15

Published by the American Institute of Physics.

\section{Additional information on J. Chem. Phys.}

Journal Homepage: http://jcp.aip.org/

Journal Information: http://jcp.aip.org/about/about_the_journal

Top downloads: http://jcp.aip.org/features/most_downloaded

Information for Authors: http://jcp.aip.org/authors

\section{ADVERTISEMENT}

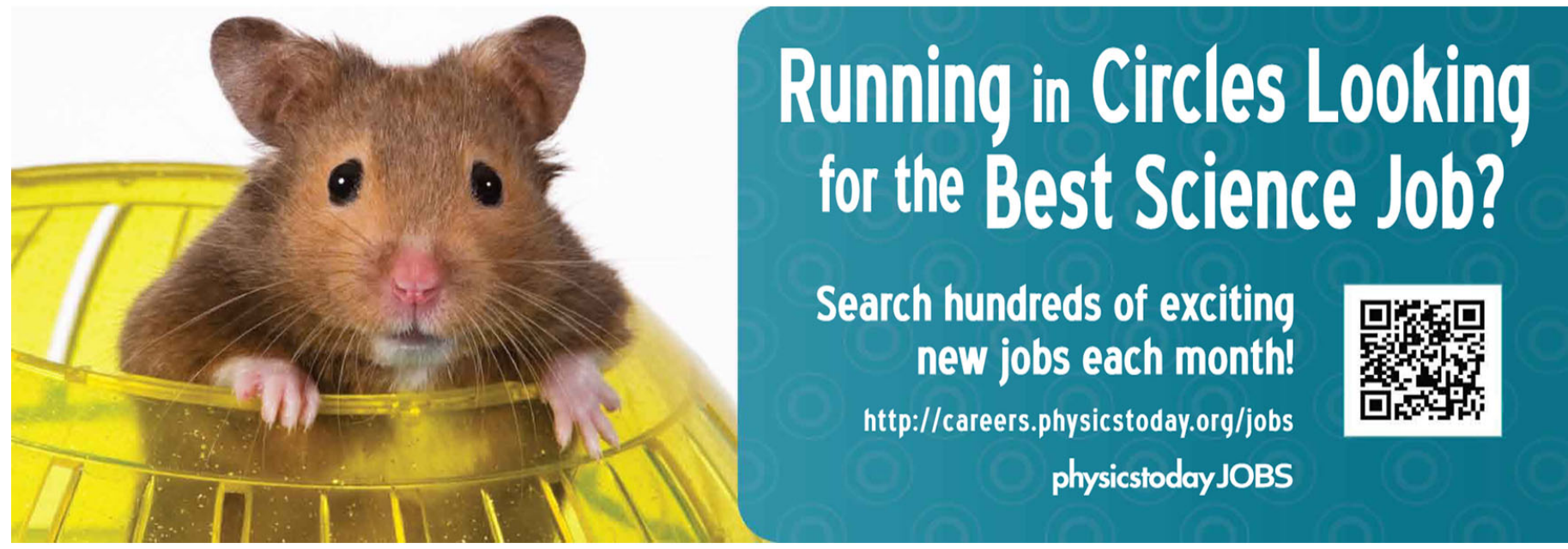




\title{
Elasticity of flexible and semiflexible polymers with extensible bonds in the Gibbs and Helmholtz ensembles
}

\author{
Fabio Manca, ${ }^{1}$ Stefano Giordano, ${ }^{2}$ Pier Luca Palla, ${ }^{2}$ Rinaldo Zucca, ${ }^{2}$ Fabrizio Cleri, ${ }^{2}$ \\ and Luciano Colombo ${ }^{1,3, a)}$ \\ ${ }^{1}$ Dipartimento di Fisica, Università di Cagliari, Cittadella Universitaria, 09042 Monserrato, Cagliari, Italy \\ ${ }^{2}$ Institute Electronique, Microélectronique et Nanotechnologie (IEMN), UMR CNRS 8520, Avenue Poincaré, \\ BP 60069, 59652, Villeneuve d'Ascq, France \\ ${ }^{3}$ IOM-CNR (Unità Cagliari), Cittadella Universitaria, 09042 Monserrato, Cagliari, Italy
}

(Received 23 December 2011; accepted 2 April 2012; published online 19 April 2012)

\begin{abstract}
Stretching experiments on single molecules of arbitrary length opened the way for studying the statistical mechanics of small systems. In many cases in which the thermodynamic limit is not satisfied, different macroscopic boundary conditions, corresponding to different statistical mechanics ensembles, yield different force-displacement curves. We formulate analytical expressions and develop Monte Carlo simulations to quantitatively evaluate the difference between the Helmholtz and the Gibbs ensembles for a wide range of polymer models of biological relevance. We consider generalizations of the freely jointed chain and of the worm-like chain models with extensible bonds. In all cases we show that the convergence to the thermodynamic limit upon increasing contour length is described by a suitable power law and a specific scaling exponent, characteristic of each model. (C) 2012 American Institute of Physics. [http://dx.doi.org/10.1063/1.4704607]
\end{abstract}

\section{INTRODUCTION}

The recent development of mechanical experiments on single molecules provided a deeper understanding of intermolecular and intramolecular forces, thereby introducing crucial additional information about the thermodynamics and kinetics of several biomolecular processes. ${ }^{1-3}$ Single-molecule experimental methods can be typically based on laser optical tweezers, ${ }^{3}$ magnetic tweezers, ${ }^{4}$ or atomic force microscope. ${ }^{5}$ These experimental techniques have been extensively applied to nucleic acids (DNA, RNA, and DNA condensation) ${ }^{6}$, proteins (protein-protein interaction ${ }^{7}$ and protein folding ${ }^{8}$ ), molecular motors, ${ }^{9}$ and other long-chain biopolymers. ${ }^{10}$ Furthermore, in such experiments the determination of small energies and the detection of large deviations due to Brownian interactions offer a new method for analysing the basic foundations of statistical mechanics. In particular, the above techniques permitted a clearer comprehension of the equilibrium and non-equilibrium thermodynamics of small systems and the experimental verification of fluctuation theorems. ${ }^{11-13}$

One of the most important single-molecule experiment concerns the stretching of a chain structure in order to measure its elastic features. The first famous investigation was performed on the double-stranded DNA and the results appeared in very good agreement with the worm-like chain (WLC) model ${ }^{14,15}$ while they were only in partial agreement with the freely jointed chain (FJC) model (the latter model typically providing, however, a better fit for single-stranded DNA and RNA (Ref. 16)). This result and successive experimental evidences suggested that the mechanical properties

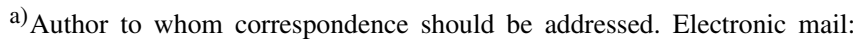
luciano.colombo@dsf.unica.it.
}

of DNA, and in particular its flexibility (described by the persistence length $\left.L_{p}\right)^{17}$, have a relevant role in many biological process. ${ }^{18}$ The importance of understanding the force-extension relationship for macromolecules has therefore attracted the attention of physicists and mathematicians, who produced several models and relationships to explain the experimental results. For example, the discrete version of the WLC model was considered and new interpolation formulas for semiflexible polymers were introduced. ${ }^{19,20}$ Moreover, efficient techniques for calculating the thermo-mechanical properties of a heterogeneous chain were developed through the numerical determination of the statistical partition functions under different types of boundary conditions. ${ }^{21,22}$

Two main assumptions are typically considered in the analytical developments of the standard FJC and WLC models: (a) inextensibility, expressed as a fixed bond length between two adjacent monomers and (b) the contour length $L_{c}$ (equal to $N$ times the equilibrium bond length for a chain composed of $N$ monomers) is supposed very large. The first assumption should not be adopted if we consider a large applied force as explained in Sec. II. The second assumption is related to the concept of thermodynamic limit. In fact, the standard rules of equilibrium thermodynamics may not apply to experiments on individual, short-length polymer molecules. In such a case the results may depend on the boundary conditions imposed for stretching the polymer, namely: a fixed end-to-end distance, pertinent to the Helmholtz ensemble of statistical mechanics, or a fixed force applied at one or both ends, rather representing a realization of the Gibbs ensemble. The differences between the two conditions should vanish in the thermodynamic limit $(N \rightarrow \infty)$, when the polymer contour length $L_{c}$ is much larger than its persistence length $L_{p}$ (or to the individual bond length, for completely flexible chains). This phenomenon has been studied and observed for simple models 
describing internal barriers to bond rotation, ${ }^{23}$ for the single ideal Gaussian chain ${ }^{24}$ and for DNA stretching. ${ }^{25,26}$

It is important to observe that from the experimental point of view both assumptions, inextensibility and attainment of thermodynamic limit, are often not fulfilled for many practical reasons (e.g., too large applied forces and force-rates, different configurations of the experimental devices, actual procedures for force and distance measurements, and so on). Therefore, in this paper we study the effects of the superposition of the two aspects, by considering the mechanical stretching response of extensible polymer chains of arbitrary length. Of course, the above two assumptions are completely independent and, therefore, one can consider all their possible combinations: short chains without extensibility (leading to the FJC or WLC for short chains), extensible long chains at the thermodynamic limit, or short chains with extensibility.

In particular, we will take into consideration the quantitative difference between the thermodynamic behaviour within either the Helmholtz or Gibbs ensemble, by investigating the foundations of the statistical mechanics for small systems. This will be done by taking into account flexible (or semiflexible) and extensible polymer chain models of biological interest. Our theoretical approach is twofold, since we adopt both analytical and numerical techniques (Monte Carlo simulations). Indeed, while the analytical approach is useful to obtain the explicit partition function in some specific cases, Monte Carlo simulations are crucial to determine the scaling laws controlling the convergence to the thermodynamic limit.

The structure of the paper is the following. In Sec. II we introduce the thermodynamics of a polymer chain by describing two different approaches, respectively, leading to the Helmholtz and the Gibbs ensembles. Then, we derive an exact relationship between the partition functions pertinent to the different ensembles. By using this result we formally prove the existence of the thermodynamic limit for long chains. In Secs. III and IV we quantitatively evaluate the difference between the Helmholtz and Gibbs ensembles for FJC and WLC models with extensible bonds. In all cases we find a convergence to the thermodynamic limit described by a suitable power law and a specific scaling exponent. Finally, in Sec. V some conclusions and perspectives are drawn.

\section{THERMODYNAMICS OF POLYMER CHAINS}

\section{A. General theoretical framework}

Usually, FJC and WLC models describe the polymer as an ideal chain, where self-avoidance is not taken into account. ${ }^{27,28}$ In both cases the polymer is modeled as a chain of beads connected by rigid bonds, but only the WLC model takes into account an intrinsic bending stiffness between two consecutive bonds. The most important common feature is the inextensibility of the bonds. Such assumption is used even in the case of large applied forces, although it has been shown that the polymer may enter a regime where the elasticity of the molecular and chemical bonds becomes important. This phenomenon has been experimentally observed for DNA, ${ }^{29}$ polyelectrolytes, ${ }^{30}$ and F-actin. ${ }^{31}$ Typical corrections aimed at describing bond elasticity have been introduced through ad- ditional spring-like terms. ${ }^{32}$ Recently, a refined statistical mechanics approach for the stretching of semiflexible polymers with elastic bonds was developed. ${ }^{33}$

Let us consider a chain of monomers in a long molecule, each monomer representing a group of atoms or molecules along the polymer backbone. The classical dynamics of monomers is described by the set of positions $\vec{r}_{i}(i$ $=1, \ldots, N)$ and momenta $\vec{p}_{i}(i=1, \ldots, N)$. The polymer end-to-end distance is defined as $\left|\vec{r}_{N}-\vec{r}_{0}\right|$ and the contour length of the polymer is defined by $L_{c}=\sum_{i=0}^{N-1}\left\langle\left|\vec{r}_{i+1}-\vec{r}_{i}\right|\right\rangle$, where average values are determined through the pertinent probability density $\rho$ (see below). We assume that one terminal monomer is fixed at position $\vec{r}_{0} \equiv(0,0,0)$ and that monomers interact through a potential $V=V\left(\vec{r}_{1}, \ldots, \vec{r}_{N}\right)$. This implies that our scheme will be able to describe all types of polymer models, ranging from the FJC to the WLC and possible generalizations with extensible bonds. The dynamics of the system is described by the Hamiltonian

$$
h_{0}\left(\vec{r}_{1}, \ldots, \vec{r}_{N}, \vec{p}_{1}, \ldots, \vec{p}_{N}\right)=\sum_{i=1}^{N} \frac{\vec{p}_{i} \cdot \vec{p}_{i}}{2 m}+V\left(\vec{r}_{1}, \ldots, \vec{r}_{N}\right) \text {. }
$$

We consider this system to be in thermal equilibrium with a reservoir at temperature $T$ and, therefore, its statistical properties are described by the canonical ensemble distribution

$$
\rho(q, p)=\frac{1}{Z} e^{-\frac{h_{0}(q, p)}{k_{B} T}},
$$

where we have introduced the canonical variables $q$ $=\left(\vec{r}_{1}, \ldots, \vec{r}_{N}\right)$ and $p=\left(\vec{p}_{1}, \ldots, \vec{p}_{N}\right)$, the Boltzmann constant $k_{B}$, and the partition function $Z$. The main goal of this section is to obtain the thermodynamics of the system when the end terminal monomer is either clamped at a fixed position $\vec{r}_{N}$ or it is subjected to a constant traction force $\vec{f}$. These two boundary conditions correspond to the constant-volume or constant-pressure configurations of an ideal gas, and are described by the Helmholtz or Gibbs statistical mechanics ensembles.

\section{B. Polymer chain with fixed end-to-end distance}

By setting a given end-to-end distance, positions $\vec{r}_{0}$ and $\vec{r}_{N}$ are fixed (see Fig. 1) and we can use the following reduced Hamiltonian:

$$
\begin{aligned}
& h\left(\vec{r}_{1}, \ldots, \vec{r}_{N-1}, \vec{p}_{1}, \ldots, \vec{p}_{N-1}, \vec{r}\right) \\
& \quad=h_{0}\left(\vec{r}_{1}, \ldots, \vec{r}_{N-1}, \vec{r}_{N}=\vec{r}, \vec{p}_{1}, \ldots, \vec{p}_{N-1}, \vec{p}_{N}=0\right) .
\end{aligned}
$$

In this case the microscopic variables are defined as $q$ $=\left(\vec{r}_{1}, \ldots, \vec{r}_{N-1}\right)$ and $p=\left(\vec{p}_{1}, \ldots, \vec{p}_{N-1}\right)$, in terms of which the system partition function is written

$$
Z_{\vec{r}}(\vec{r}, T)=\iint_{\Gamma_{N-1}} e^{-\frac{h(q, p, \vec{r})}{k_{B} T}} d q d p,
$$

where $\Gamma_{N-1}=\mathfrak{R}^{6(N-1)}$. The equilibrium distribution is therefore

$$
\rho_{\vec{r}}(q, p ; \vec{r}, T)=\frac{1}{Z_{\vec{r}}(\vec{r}, T)} e^{-\frac{h(q, p, \vec{r})}{k_{B} T}} .
$$




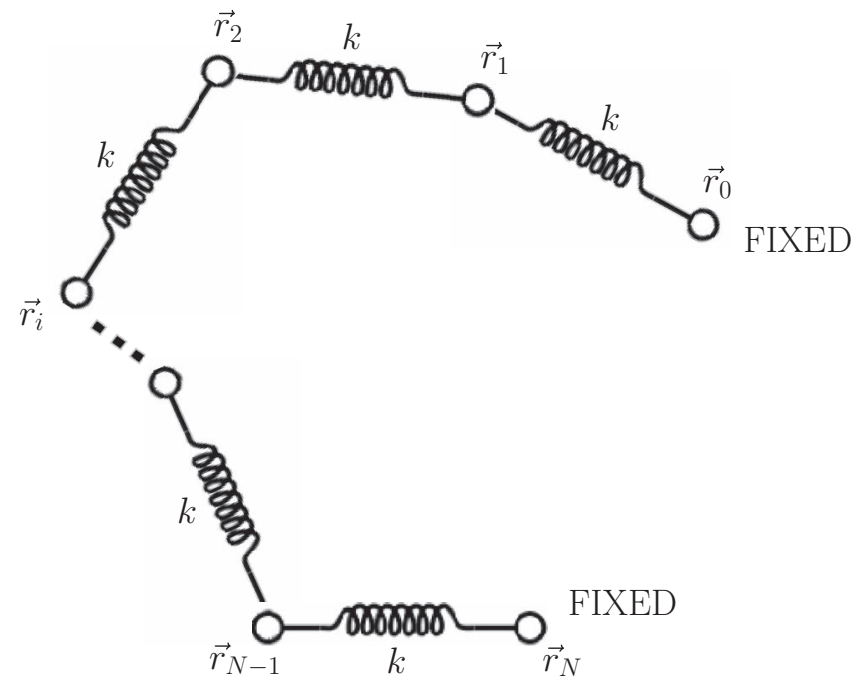

FIG. 1. A polymer chain under the Helmholtz boundary conditions: monomers $\vec{r}_{0}$ and $\vec{r}_{N}$ are clamped.

The net force exerted on the monomer at position $\vec{r}$ by the remaining monomers is by definition $-\frac{\partial h}{\partial \vec{r}}$. Such a force can be used to define the mechanical constitutive equation (hereafter referred as the "force-extension curve") of the chain. In fact, the force exerted on the system (from the outside) in order to keep fixed the last monomer is $\vec{f}=\left\langle\frac{\partial h}{\partial \vec{r}}\right\rangle$, thus providing the statistical nonlinear generalization of Hooke's law for the chain

$$
\vec{f}(\vec{r}, T)=-k_{B} T \frac{\partial}{\partial \vec{r}} \log Z_{\vec{r}}=\frac{\partial F(\vec{r}, T)}{\partial \vec{r}},
$$

where $F(\vec{r}, T)=-k_{B} T \log Z_{\vec{r}}$ is the Helmholtz free energy. We identify the average value of the Hamiltonian as the internal energy of the system $U=\langle h(q, p, \vec{r})\rangle$. In order to introduce the thermodynamics we must consider a transformation of the system, i.e., a process which imposes a time-dependent variation of some macroscopic variable of the system. This means that the end-position $\vec{r}=\vec{r}(t)$ can vary in time as well as the temperature $T=T(t)$. By assuming slow variations of $\vec{r}$ and $T$ the system undergoes a quasi-static transformation for which the distribution given in Eq. (5) is valid at any time. We define the entropy

$$
S_{\vec{r}}=-k_{B}\left\langle\log \rho_{\vec{r}}\right\rangle=-k_{B} \iint_{\Gamma_{N-1}} \rho_{\vec{r}} \log \rho_{\vec{r}} d q d p,
$$

and we easily get the thermodynamic energy balance in the form $^{34,35}$

$$
\frac{d U}{d t}=\vec{f} \cdot \frac{d \vec{r}}{d t}+T \frac{d S_{\vec{r}}}{d t},
$$

where the first term represents the work done by the external force and the second one represents the heat entering the system. Finally, the Helmholtz free energy $F$ is given by $F=U-T S_{\vec{r}}$

\section{Polymer chain under constant load}

We next assume that a given force $\vec{f}$ is applied to the terminal monomer at $\vec{r}_{N}$, while the end-to-end distance is free

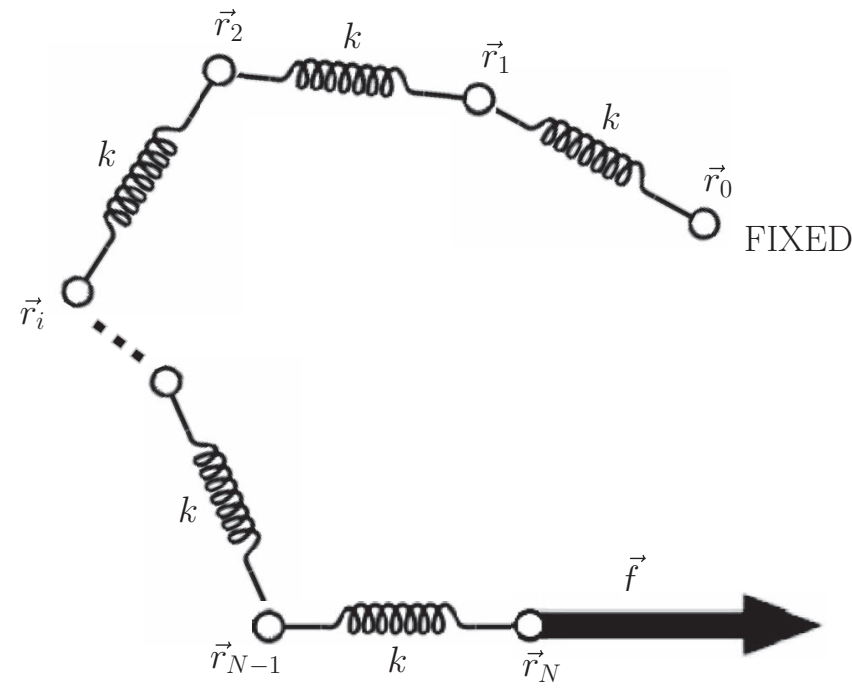

FIG. 2. A polymer chain under the Gibbs boundary conditions: while the monomer $\vec{r}_{0}$ is clamped, the one at $\vec{r}_{N}$ is subject to a constant traction $\vec{f}$.

to fluctuate (see Fig. 2). This external force is described by an additional potential energy term given by $-\vec{f} \cdot \vec{r}_{N}$ since $-\frac{\partial}{\partial \vec{r}_{N}}\left(-\vec{f} \cdot \vec{r}_{N}\right)=\vec{f}$. Therefore, the system is described by the following augmented Hamiltonian:

$$
\begin{aligned}
& \widetilde{h}\left(\vec{r}_{1}, \ldots, \vec{r}_{N}, \vec{p}_{1}, \ldots, \vec{p}_{N}, \vec{f}\right) \\
& \quad=h_{0}\left(\vec{r}_{1}, \ldots, \vec{r}_{N}, \vec{p}_{1}, \ldots, \vec{p}_{N}\right)-\vec{f} \cdot \vec{r}_{N},
\end{aligned}
$$

where $q=\left(\vec{r}_{1}, \ldots, \vec{r}_{N}\right)$ and $p=\left(\vec{p}_{1}, \ldots, \vec{p}_{N}\right)$ are the microscopic variables and $\vec{f}$ acts as a macroscopic variable. The ensemble partition function is now given by

$$
Z_{\vec{f}}(\vec{f}, T)=\iint_{\Gamma_{N}} e^{-\frac{\tilde{T}(q, p, \vec{f})}{k_{B} T}} d q d p,
$$

where $\Gamma_{N}=\mathfrak{R}^{6 N}$ and the corresponding statistical distribution is

$$
\rho_{\vec{f}}(q, p ; \vec{f}, T)=\frac{1}{Z_{\vec{f}}(\vec{f}, T)} e^{-\frac{\tilde{h}(q, p, \vec{f})}{k_{B} T}} .
$$

We observe that $\frac{\partial \widetilde{h}}{\partial \vec{f}}=-\vec{r}_{N}$ and we calculate the mean position of the last monomer of the chain through the average value $\vec{r}=\left\langle\vec{r}_{N}\right\rangle$ or, more explicitly, $\vec{r}=-\left\langle\frac{\partial \widetilde{h}}{\partial \vec{f}}\right\rangle$. This constitutive equation can also be expressed in terms of the partition function $Z_{\vec{f}}$. In fact, by differentiating Eq. (10) with respect to $\vec{f}$, we get

$$
\vec{r}(\vec{f}, T)=k_{B} T \frac{\partial}{\partial \vec{f}} \log Z_{\vec{f}}=-\frac{\partial G(\vec{f}, T)}{\partial \vec{f}},
$$

where $G(\vec{f}, T)=-k_{B} T \log Z_{\vec{f}}$ is the Gibbs free energy. We now introduce the thermodynamics by identifying the average value of the augmented Hamiltonian with the enthalpy of the system $H=\langle\widetilde{h}(q, p, \vec{f})\rangle$. As before, we assume slow variations $\vec{f}=\vec{f}(t)$ and $T=T(t)$ in order to deal with a quasi-static transformation and we develop the time derivative of the enthalpy. Through the new definition of the entropy

$$
S_{\vec{f}}=-k_{B}\left\langle\log \rho_{\vec{f}}\right\rangle=-k_{B} \iint_{\Gamma_{N}} \rho_{\vec{f}} \log \rho_{\vec{f}} d q d p,
$$


the energy balance is obtained as 34,35

$$
\frac{d H}{d t}=-\frac{d \vec{f}}{d t} \cdot \vec{r}+T \frac{d S_{\vec{f}}}{d t}
$$

It is important to remark that Eq. (13) is not identical to Eq. (7) since they are based on two different probability densities. Only when the thermodynamic limit $(N \rightarrow \infty)$ is attained, the two approaches would yield the same results (see Sec. II E for details). From the previous expression we also obtain that $G=H-T S_{\vec{f}}$, according to the standard definition of the Gibbs free energy.

\section{Helmholtz versus Gibbs ensembles}

By taking into consideration Eqs. (4) and (10), as well as the known integral

$$
\int_{\mathfrak{R}^{3}} \exp \left\{-\frac{1}{k_{B} T} \frac{\vec{p} \cdot \vec{p}}{2 m}\right\} d \vec{p}=\left(2 \pi m k_{B} T\right)^{3 / 2},
$$

the exact relationship between $Z_{\vec{f}}$ and $Z_{\vec{r}}$ can be obtained

$$
Z_{\vec{f}}(\vec{f}, T)=\left(2 \pi m k_{B} T\right)^{\frac{3}{2}} \int_{\mathfrak{R}^{3}} Z_{\vec{r}}(\vec{r}, T) e^{\frac{\vec{f} \cdot \vec{r}}{k_{B} T}} d \vec{r},
$$

showing that the Gibbs partition function is the threedimensional (bilateral or two-sided) Laplace transform of the Helmholtz partition function (except for a non relevant multiplicative constant).

For the following purposes, it is useful to invert the previous integral relation. In Eq. (16) we operate the change of variable $\vec{f}=-i k_{B} T \vec{\omega}$ (with $\vec{\omega} \in \mathfrak{R}^{3}$ ), so that

$$
\frac{Z_{\vec{f}}\left(-i k_{B} T \vec{\omega}, T\right)}{\left(2 \pi m k_{B} T\right)^{\frac{3}{2}}}=\int_{\mathfrak{R}^{3}} Z_{\vec{r}}(\vec{r}, T) e^{-i \vec{\omega} \cdot \vec{r}} d \vec{r} .
$$

In the right-hand side we recognize a standard Fourier transform and the inverse relation is obtained as

$$
Z_{\vec{r}}(\vec{r}, T)=\frac{1}{(2 \pi)^{3}} \int_{\mathfrak{R}^{3}} \frac{Z_{\vec{f}}\left(-i k_{B} T \vec{\omega}, T\right)}{\left(2 \pi m k_{B} T\right)^{\frac{3}{2}}} e^{i \vec{\omega} \cdot \vec{r}} d \vec{\omega} .
$$

By the change of variable $\vec{\phi}=-k_{B} T \vec{\omega}$ we obtain the relation $d \vec{\phi}=\left(k_{B} T\right)^{3} d \vec{\omega}$ and, therefore, we get

$$
Z_{\vec{r}}(\vec{r}, T)=\frac{1}{\left[\left(2 \pi k_{B} T\right)^{3} m\right]^{3 / 2}} \int_{\mathfrak{R}^{3}} Z_{\vec{f}}(i \vec{\phi}, T) e^{-i \frac{\vec{\phi} \cdot \vec{r}}{k_{B} T}} d \vec{\phi}
$$

which states that, in order to derive the Helmholtz partition function, one must use the analytic continuation of the Gibbs partition function over the imaginary argument.

Equations (16) and (19) allow to obtain each partition function with an integral over $\mathfrak{R}^{3}$, which is much easier than the original integral over the whole phase space. So, when one of the two partition functions is determined, the other can be simply obtained analytically or numerically, depending on the complexity of the system. Moreover, the relationship between the two partition functions is very important for enlightening the meaning of the thermodynamic limit, as described below.

The present results can be further simplified when the applied force is taken to be collinear with the end-to-end vector distance. We suppose that the Helmholtz partition function exhibits the spherical symmetry, leading to the scalar relation $Z_{\vec{r}}(\vec{r}, T)=Z_{r}(r, T)$. In other words, $Z_{\vec{r}}$ depends on $\vec{r}$ only through its modulus $r$. In this case the Gibbs partition function shows such a spherical symmetry as well: $Z_{\vec{f}}(\vec{f}, T)=Z_{f}(f, T)$. Conversely, a similar constraint is, in turn, obtained for the Helmoltz function if spherical symmetry is assumed for the Gibbs function. It can be proved that $Z_{r}$ and $Z_{f}$ fulfil the following relationships:

$$
Z_{f}(f, T)=\alpha \int_{0}^{\infty} Z_{r}(r, T) \frac{\beta r}{f} \sinh \frac{f r}{k_{B} T} d r
$$

and

$$
Z_{r}(r, T)=\alpha \int_{0}^{\infty} Z_{f}(i \eta, T) \frac{\eta}{\beta r} \sin \frac{\eta r}{k_{B} T} d \eta,
$$

where $\alpha=\left(\frac{2}{\pi k_{B} T}\right)^{1 / 2}$ and $\beta=\frac{\left(2 \pi m k_{B} T\right)^{3}}{m^{3 / 2}}$. We observe that Eqs. (20) and (21) are the counterparts of Eqs. (16) and (19) for a model with spherical symmetry.

\section{E. On the thermodynamic limit}

We want to prove that the Helmholtz and Gibbs ensembles provide the same constitutive equation for large systems $(N \rightarrow \infty)$. The two expressions given in Eqs. (6) and (12) would represent, in fact, the same constitutive equation if the first relation coincides with the inverse function of the second one and vice versa. In other words, our statement above is proved as long as it is shown that the Helmholtz and the Gibbs energy functions are related by a Legendre transformation $G=F-\vec{f} \cdot \vec{r}$, as discussed in Ref. 35. By inserting Eqs. (6) and (12) in Eq. (16) we obtain

$$
e^{-\frac{G(\vec{f}, T)}{k_{B} T}}=\left(2 \pi m k_{B} T\right)^{\frac{3}{2}} \int_{\mathfrak{R}^{3}} e^{-\frac{F\left(\vec{r}^{\prime}, T\right)}{k_{B} T}} e^{\frac{\vec{f} \cdot \vec{r}^{\prime}}{k_{B} T}} d \vec{r}^{\prime},
$$

which is an exact relation between $F$ and $G$, always satisfied (i.e., valid for any $N)$. By expanding $F(\vec{r}, T)$ up to the second order in $\vec{r}$,

$$
\left.\left.F\right|_{\vec{r}^{\prime}} \simeq F\right|_{\vec{r}}+\frac{\partial F}{\partial \vec{r}}\left(\vec{r}^{\prime}-\vec{r}\right)+\frac{1}{2}\left(\vec{r}^{\prime}-\vec{r}\right) \cdot \frac{\partial^{2} F}{\partial \vec{r}^{2}}\left(\vec{r}^{\prime}-\vec{r}\right) .
$$

In the previous series expansion we did not consider the third order term and higher since, for large $N$, their effects are negligible with respect to the leading terms (first and second order). This is a typical approximation adopted and justified within the Laplace method useful for obtaining the asymptotic behavior of integrals (e.g., used for proving the standard Stirling approximation for the factorial function, largely used in several statistical mechanics evaluations). ${ }^{36}$ By means of Eq. (6) we eventually obtain

$$
G=F-\vec{f} \cdot \vec{r}-k_{B} T \ln \Gamma
$$

where

$$
\Gamma=\left(2 \pi m k_{B} T\right)^{\frac{3}{2}} \int_{\mathfrak{R}^{3}} e^{-\frac{1}{2 k_{B} T}\left(\vec{r}^{\prime}-\vec{r}\right) \frac{\partial^{2} F}{\partial \vec{r}^{2}}\left(\vec{r}^{\prime}-\vec{r}\right)} d \vec{r}^{\prime} .
$$

The quantities $G, F, \Gamma$, and $\vec{f} \cdot \vec{r}$ assume an extensive character (i.e., they are proportional to $N$ ) and, therefore, the logarithmic term in Eq. (24) becomes negligible for large systems. Thus, for $N \rightarrow \infty$, the Legendre transformation is fulfilled. In Secs. III-V we will quantitatively address the convergence 


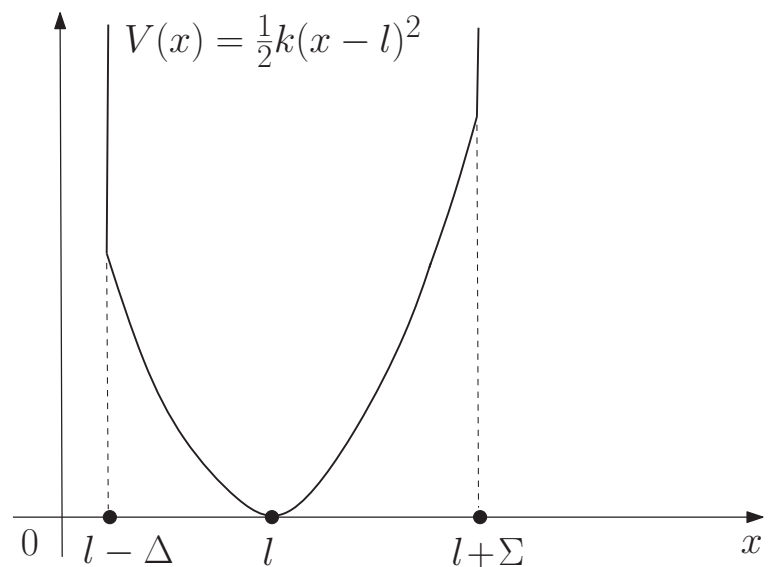

FIG. 3. Potential energy function for the stretching of a single bond in the polymer chain.

to the thermodynamic limit by comparing the Helmholtz and Gibbs ensembles for increasing values of $N$, and we will evaluate their differences for small systems (i.e., for short length polymer chains).

\section{FLEXIBLE POLYMER WITH ELASTIC BONDS}

We firstly consider a model in which each bond is represented by a harmonic spring with finite extension, while no potential is acting on bending or torsional degrees of freedom. Each spring is defined by the potential energy $V(x)$ $=(1 / 2) k(x-l)^{2}$ for $x \in(l-\Delta, l+\Sigma)$, where $k$ is the spring constant, $l$ is the equilibrium bond length, and $x$ is the actual extension of the bond. As it can be seen in Fig. 3, the potential is set to infinity for $x \notin(l-\Delta, l+\Sigma)$ in order to impose a limited extension of the spring both for expansion and compression. This assumption is consistent with that discussed in Ref. 37 , where it is shown that a strong force may induce structural transitions or even the breaking of the polymer under tension. Since such phenomena are outside the scope of this work, we limit the model to the regime of harmonic bond potentials.

We start with the Gibbs ensemble and we consider the augmented Hamiltonian

$$
\begin{aligned}
& \widetilde{h}\left(\vec{r}_{1}, \ldots, \vec{r}_{N}, \vec{p}_{1}, \ldots, \vec{p}_{N}, \vec{f}\right) \\
& \quad=\sum_{i=1}^{N} \frac{\vec{p}_{i} \cdot \vec{p}_{i}}{2 m}+\frac{1}{2} k \sum_{i=0}^{N-1}\left(\left\|\vec{r}_{i+1}-\vec{r}_{i}\right\|-l\right)^{2}-\vec{f} \cdot \vec{r}_{N}
\end{aligned}
$$

In this case the determination of the spherically symmetric partition function can be made in closed form. First of all, we separate the kinetic part of the partition function and we adopt the change of variables $\vec{\xi}_{i+1}=\vec{r}_{i+1}-\vec{r}_{i}(i=0, \ldots, N-1)$. Further, by representing the vector $\vec{\xi}_{i}$ in spherical coordinates, we get

$$
\begin{aligned}
Z_{f}(f, T)= & \left(\sqrt{2 \pi m k_{B} T}\right)^{3 N}\left(\frac{4 \pi k_{B} T}{f}\right)^{N} \\
& \times\left[\int_{l-\Delta}^{l+\Sigma} \exp \left\{-\frac{k}{2 k_{B} T}(\xi-l)^{2}\right\} \sinh \left(\frac{f \xi}{k_{B} T}\right) \xi d \xi\right]^{N}
\end{aligned}
$$

The calculation can be explicitly carried out by making use of the known integral

$$
\begin{aligned}
I\left(\alpha, \beta, x_{0}, a, b\right)= & \int_{a}^{b} x e^{-\alpha\left(x-x_{0}\right)^{2}} e^{\beta x} d x \\
= & e^{\beta x_{0}} e^{\frac{\beta^{2}}{4 \alpha}}\left\{\frac{1}{2 \alpha}\left(e^{-\mathcal{A}^{2}}-e^{-\mathcal{B}^{2}}\right)\right. \\
& \left.+\sqrt{\frac{\pi}{\alpha}} \frac{\beta+2 \alpha x_{0}}{4 \alpha}[\operatorname{erf}(\mathcal{B})-\operatorname{erf}(\mathcal{A})]\right\},
\end{aligned}
$$

where

$$
\mathcal{A}=\sqrt{\alpha}\left(a-x_{0}-\frac{\beta}{2 \alpha}\right), \mathcal{B}=\sqrt{\alpha}\left(b-x_{0}-\frac{\beta}{2 \alpha}\right),
$$

and the function $\operatorname{erf}(z)$ is defined $\operatorname{as}^{38}$

$$
\operatorname{erf}(z)=\frac{2}{\sqrt{\pi}} \int_{0}^{z} e^{-t^{2}} d t
$$

From Eq. (28) we can further define the following auxiliary function:

$$
\Pi(\alpha, \beta, x, a, b)=I(\alpha, \beta, x, a, b)-I(\alpha,-\beta, x, a, b) .
$$

The calculation leads to the exact expression of the Gibbs partition function in the form

$$
\begin{aligned}
Z_{f}(f, T)= & \left(\sqrt{2 \pi m k_{B} T}\right)^{3 N}\left(\frac{2 \pi k_{B} T}{f}\right)^{N} \\
& \times\left[\Pi\left(\frac{k}{2 k_{B} T}, \frac{f}{k_{B} T}, l, l-\Delta, l+\Sigma\right)\right]^{N} .
\end{aligned}
$$

This partition function directly provides the (scalar) constitutive equation $r=r(f)$ as

$$
r=k_{B} T \frac{\partial \log Z_{f}(f, T)}{\partial f} .
$$

It is interesting to observe that, as expected, in the limit of $k$ $\rightarrow \infty$ the partition function of the FJC model is recovered

$$
Z_{f}(f, T)=\text { cost. } \times\left(\frac{\sinh \left(\frac{l f}{k_{B} T}\right)}{\frac{l f}{k_{B} T}}\right)^{N}
$$

and the constitutive equation $r=N l \mathcal{L}\left(\frac{l f}{k_{B} T}\right)$ is found, where $\mathcal{L}(x)=\operatorname{coth} x-1 / x$ is the Langevin function. ${ }^{35,39}$

In Fig. 4 we plot the constitutive equations (at constant temperature $T=293 K$ ) obtained by applying Eqs. (32) and (33). We adopted the parameters $l=2.5 \mathrm{~nm}$ (typically used for DNA (Refs. 19 and 20)), placing the walls at $l-\Delta=0$ and $\Sigma+l=5 \mathrm{~nm}$. The plot represents elongation versus traction curves for different values of the spring constant $k=3,5,10,20,50,2000$ in units of $k_{B} T /(\mathrm{nm})^{2}$ $=4 \times 10^{-3} \mathrm{~N} / \mathrm{m}$. While the smallest value of the stretching modulus describes a very soft chain, the largest one actually mimics a FJC. Three different regimes can be detected in Fig. 4: the first one, corresponding to a very small applied force, is the entropic region characterized by a linear relation 


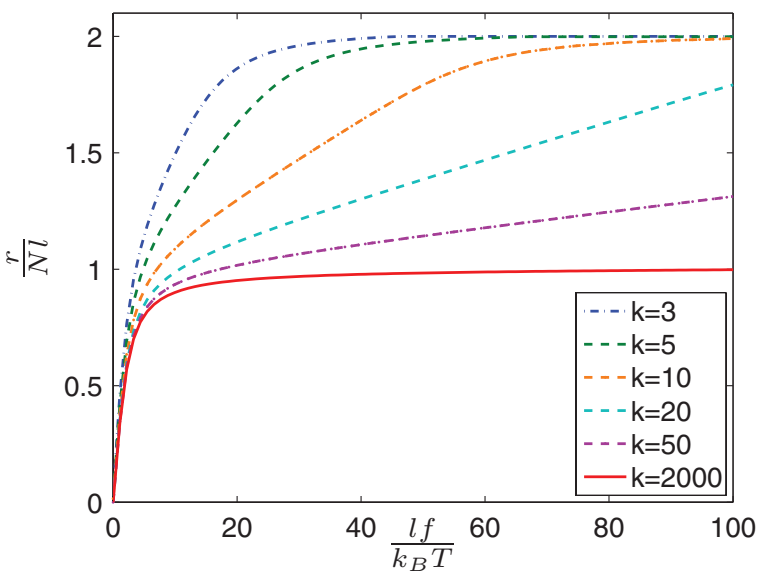

FIG. 4. Constitutive relation provided by Eq. (33) for a flexible polymer with elastic bonds described by the potential reported in Fig. 3. The elongation $r$ and the traction $f$ are reported, respectively, in the vertical and horizontal axis in dimensionless units (see text). Different relations in the Gibbs ensemble are reported, corresponding to a spring constant varying in the range $3 \leq k$ $\leq 2000$ (units of $4 \times 10^{-3} \mathrm{~N} / \mathrm{m}$ ).

$r=N l^{2} f /\left(3 k_{B} T\right)$, as it is well known from classical polymer theory $; 3,39$ by increasing the applied load, the chain experiences an elastic region characterized by a slope proportional to $k$; finally, the saturation is reached when the bond extension approaches the energy barrier at $l+\Sigma$. The length of the polymer chain in the configuration of largest extension $(f \rightarrow \infty)$ is $N(l+\Sigma)$. It is important to remark that each curve is independent of $N$, since the partition function given in Eq. (32) is an exact power with exponent $N$.

For the Helmholtz ensemble we use instead Eq. (21) combined with the analytic continuation of Eq. (32) with $f=i \eta$. From this equation we obtain the scalar constitutive equation $f=f(r)$ (see Eq. (6))

$$
f=-k_{B} T \frac{\partial \log Z_{r}(r, T)}{\partial r},
$$

representing the elastic behaviour in the Helmholtz ensemble which depends on the number of monomers $N$. We expect a family of curves approaching the Gibbs solution for large $N$ (thermodynamic limit).

While the numerical implementation of Eq. (33) is straightforward, the Helmholtz case (see Eq. (35)) is more difficult to handle because of some numerical instabilities and it can be used in a limited range of cases. Therefore, we exploited a Monte Carlo approach which can bypass such problems and can be also adopted for studying more complex chains, such as the WLC model.

The Monte Carlo approach simulates the stretching of the polymer under a force provided by a cantilever (mimiking, for instance, the loading by an atomic force microscope) with a proper adjustable elastic stiffness. In the limit of a soft cantilever the generalized ensemble of the coupled molecule/cantilever system reduces to the Gibbs ensemble for the isolated molecule subjected to a constant force. On the other hand, for a stiff cantilever we obtain the Helmholtz ensemble for the isolated molecule held at a fixed extension by the fluctuating force. ${ }^{40}$ This simulation protocol has been already used to prove the existence of transitions from the flexi-

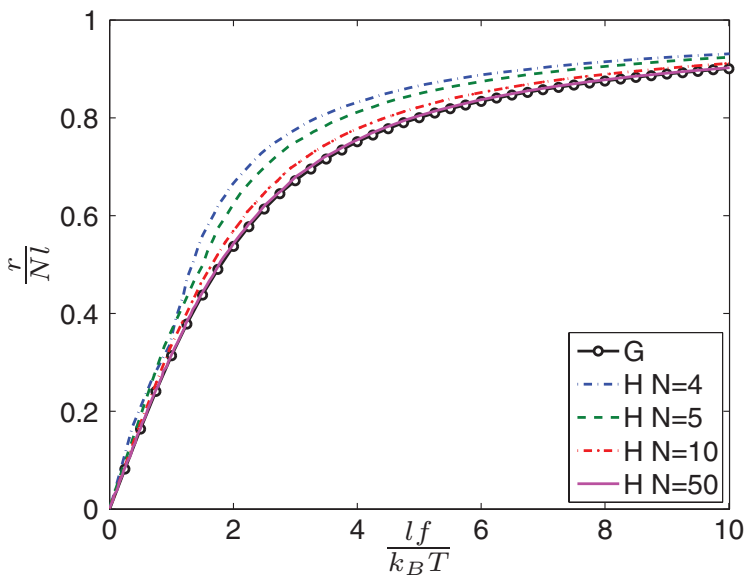

FIG. 5. Constitutive relation provided by Monte Carlo simulations for a FJC model, both under Helmholtz $(H)$ and Gibbs $(G)$ boundary conditions. The elongation $r$ and the traction $f$ are reported, respectively, on the vertical and horizontal axis in dimensionless units (see text). The Helmholtz constitutive relation is reported for different polymer lengths, given by the number $N$ of monomers in the chain.

ble to the rigid phase for the WLC model under the Helmholtz ensemble. ${ }^{41,42}$

Simulations were performed by using a rather conventional implementation of the Metropolis version of the Monte Carlo algorithm. ${ }^{43}$ The initial state of the chain is defined by a set of randomly chosen positions for the monomers. The displacement extent $\delta \vec{r}_{i}$ of the position vectors governs the magnitude of the trial move and the overall efficiency of the configurational space sampling. However, while a larger $\delta \vec{r}_{i}$ could speed up the search for the minimum, a too large $\delta \vec{r}_{i}$ leads to a high rejection frequency. Therefore, we analysed several runs in order to optimize its value. ${ }^{44,45}$ We avoided the dynamical adjustment of this parameter since this approach can violate the detailed balance. ${ }^{46}$

In Fig. 5 we report our Monte Carlo results for the FJC model in both statistical mechanics ensembles. While we observe a single curve for the Gibbs ensemble (since the Langevin function derived from Eq. (34) is independent of $N$ ), we get different elastic response curves for the Helmholtz ensemble with polymer length $N=4,5,10$, and 50 . It is interesting to note that the Helmholtz curves approach the Gibbs one for large $N$, as expected for the convergence to the thermodynamic limit. Figure 5 shows that differences between the two ensembles are considerable for short chains.

In order to better characterize the convergence toward the thermodynamic limit, we investigate the ratio between the elongation $r_{H}(N)=r /(N l)$ calculated in the Helmholtz ensemble and the elongation $r_{G}=r /(N l)$ for the Gibbs ensemble corresponding to the same number of monomers. Figure 6 proves that Monte Carlo simulations are nicely fitted by the power law

$$
\frac{r_{H}(N)}{r_{G}}=1+\frac{a}{N^{\alpha}}
$$

where $a$ and $\alpha$ are fitting parameters. Each curve corresponds to a different value of the normalized force $f l\left(k_{B} T\right)$. All data sets were interpolated by linear regression and they provided the same scaling exponent $\alpha=1.15 \pm 0.05$. Therefore, we 


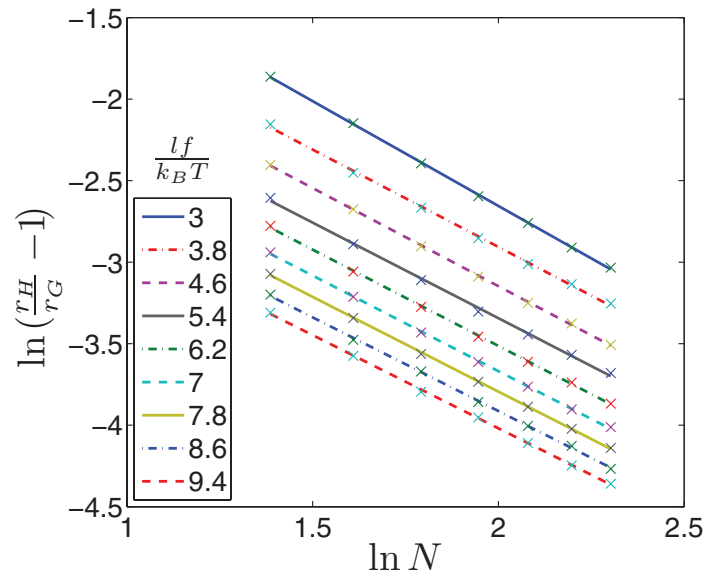

FIG. 6. Comparison between the elongations $r_{H}$ and $r_{G}$ for the pure FJC model. The elongations are calculated, respectively, in the Helmholtz and Gibbs ensembles as a function of the polymer lengths (given by the number $N$ of monomers in the chain). All sets of data are nicely fitted by Eq. (36) with the same scaling exponent $\alpha=1.15 \pm 0.05$.

argue that the convergence to the thermodynamic limit of the FJC model is quantitatively controlled by a unique scaling exponent.

Further, we are interested in understanding whether this value depends on the specific microscopic model of polymer adopted for the Monte Carlo simulations. To this aim we set a lower value for the elastic constant of the spring between the monomers. In particular, we selected the value $k=10 k_{B} T /(\mathrm{nm})^{2}$ already used in Fig. 4. The Monte Carlo simulation results for both the Helmholtz and Gibbs ensemble are shown in Fig. 7. As before, we find a single curve for the Gibbs ensemble and a family of curves for the Helmholtz ensemble (corresponding to $N=4,5,10$, and 50, from the top to the bottom). We checked and confirmed the agreement between Eq. (35) and the Monte Carlo results also for the Helmholtz ensemble. Once again, the curves in Fig. 7 can be used to address the thermodynamic limit issue, as shown in

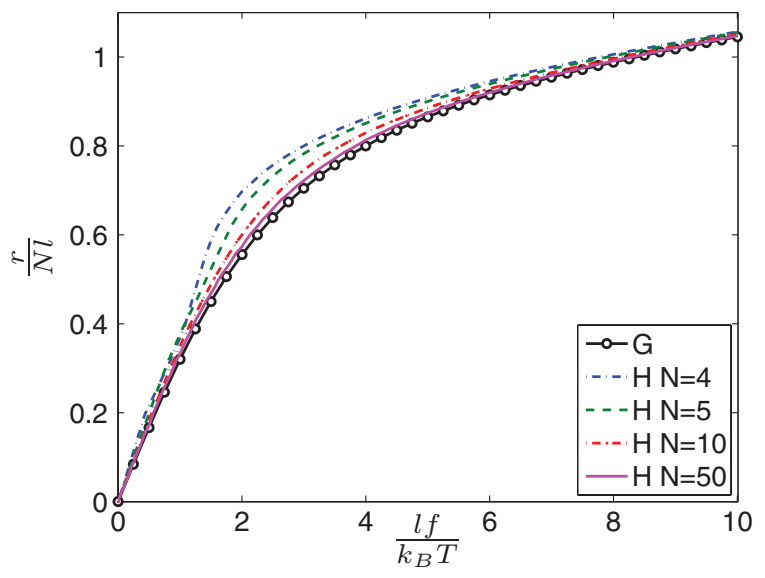

FIG. 7. Constitutive relation provided by Monte Carlo simulations for a FJC model with elastic bonds between the monomers, both under Helmholtz $(H)$ and Gibbs $(G)$ boundary conditions. The elongation $r$ and the traction $f$ are reported, respectively, on the vertical and horizontal axis in dimensionless units (see text). The Helmholtz constitutive relation is reported for different polymer lengths, given by the number $N$ of monomers in the chain.

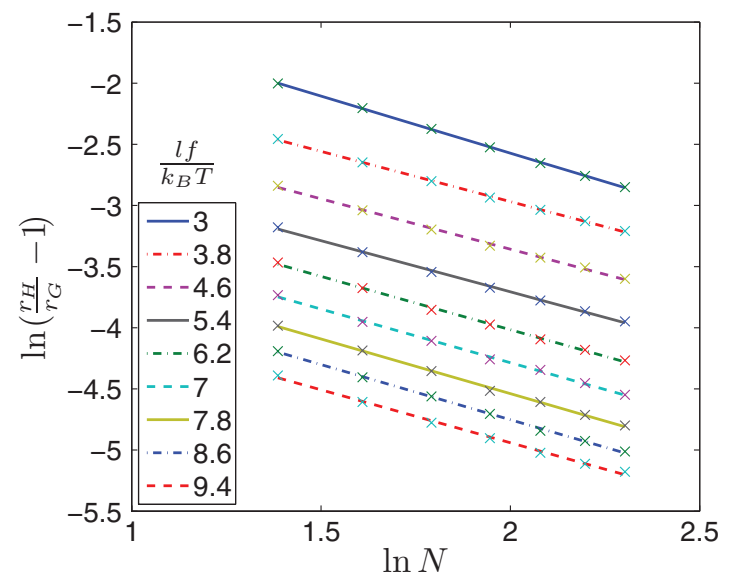

FIG. 8. Comparison between the elongations $r_{H}$ and $r_{G}$ for the FJC model with elastic bonds between the monomers. The elongations are calculated, respectively, in the Helmholtz and Gibbs ensembles as a function of the polymer length (given by the number $N$ of monomers in the chain). All sets of data are nicely fitted by Eq. (36) with the same scaling exponent $\alpha=0.80 \pm 0.05$

Fig. 8. We observe that the linear regression leads now to the scaling exponent $\alpha=0.80 \pm 0.05$ for any value of the normalized force $f l\left(k_{B} T\right)$. This result suggests that different polymer models, e.g., rigid versus elastic in this case, have different scaling exponents or, in other words, that they progress differently to reach the thermodynamic limit.

\section{SEMIFLEXIBLE POLYMER WITH ELASTIC BONDS}

In order to make the model polymer more physically sound, we now extend our formal device to a semiflexible chain which incorporates elastic bonds into a discrete version of the WLC model. The augmented Hamiltonian for the Gibbs ensemble is

$$
\begin{aligned}
& \widetilde{h}\left(\vec{r}_{1}, \ldots, \vec{r}_{N}, \vec{p}_{1}, \ldots, \vec{p}_{N}, \vec{f}\right) \\
& =\sum_{i=1}^{N} \frac{\vec{p}_{i} \cdot \vec{p}_{i}}{2 m}-\vec{f} \cdot \vec{r}_{N}+\frac{1}{2} \kappa \sum_{i=1}^{N-1}\left(\vec{t}_{i+1}-\vec{t}_{i}\right)^{2} \\
& \quad+\frac{1}{2} k \sum_{i=0}^{N-1}\left(\left\|\vec{r}_{i+1}-\vec{r}_{i}\right\|-l\right)^{2},
\end{aligned}
$$

where $\kappa$ is the bending modulus, $k$ is the stretching modulus, and $\vec{t}_{i}=\left(\vec{r}_{i+1}-\vec{r}_{i}\right) /\left\|\vec{r}_{i+1}-\vec{r}_{i}\right\|$ is the unit vector collinear with the $i$ th bond. The corresponding reduced Hamiltonian for the Helmholtz ensemble is given by

$$
\begin{aligned}
& h\left(\vec{r}_{1}, \ldots, \vec{r}_{N-1}, \vec{p}_{1}, \ldots, \vec{p}_{N-1}, \vec{r}\right) \\
& =\sum_{i=1}^{N-1} \frac{\vec{p}_{i} \cdot \vec{p}_{i}}{2 m}+\frac{1}{2} \kappa \sum_{i=1}^{N-1}\left(\vec{t}_{i+1}-\vec{t}_{i}\right)^{2} \\
& \quad+\frac{1}{2} k \sum_{i=0}^{N-1}\left(\left\|\vec{r}_{i+1}-\vec{r}_{i}\right\|-l\right)^{2},
\end{aligned}
$$

where the position of the last monomer $\vec{r}_{N}$ corresponds to the end-to-end vector $\vec{r}$. As it is well known, ${ }^{15}$ it is not possible to determine the partition functions in closed form 


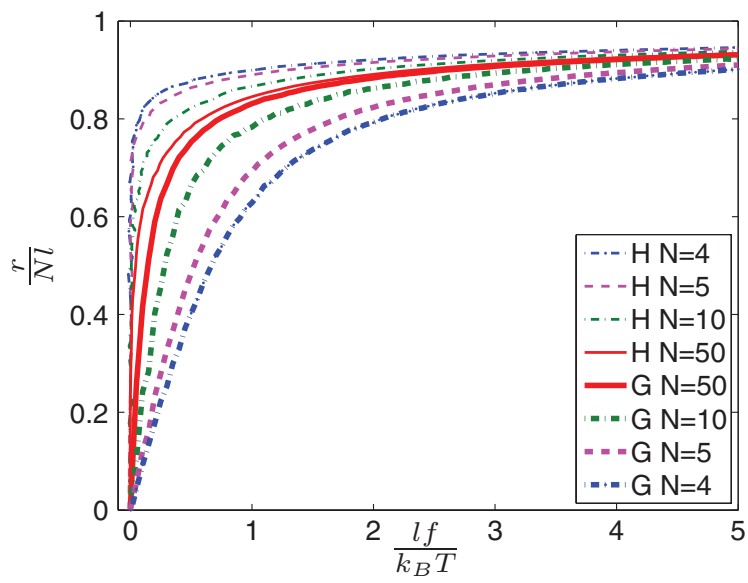

FIG. 9. Constitutive relation provided by Monte Carlo simulations for the pure WLC model, both under Helmholtz $(H)$ and Gibbs $(G)$ boundary conditions. The elongation $r$ and the traction $f$ are reported, respectively, on the vertical and horizontal axis in dimensionless units (see text). The Helmholtz constitutive relation is reported for different polymer lengths, given by the number $N$ of monomers in the chain.

for ensembles described by Eqs. (37) and (38). Therefore, we take full profit from our Monte Carlo approach and we critically address some approximated solutions. In particular, by considering an inextensible WLC model, the Hamiltonian function in the Gibbs ensemble is given by

$$
\widetilde{h}=\sum_{i=1}^{N} \frac{\vec{p}_{i} \cdot \vec{p}_{i}}{2 m}-\vec{f} \cdot \vec{r}_{N}+\frac{1}{2} \kappa \sum_{i=1}^{N-1}\left(\vec{t}_{i+1}-\vec{t}_{i}\right)^{2}
$$

while in the Helmholtz ensemble it is

$$
h=\sum_{i=1}^{N-1} \frac{\vec{p}_{i} \cdot \vec{p}_{i}}{2 m}+\frac{1}{2} \kappa \sum_{i=1}^{N-1}\left(\vec{t}_{i+1}-\vec{t}_{i}\right)^{2} .
$$

We remark that we have set the spring constant $k$ to such a very large value that the bond length remains fixed at the value $l$. The constitutive equations are now expected to depend on the number of monomers $N$ in any ensemble since, for the WLC model, both partition functions given in Eqs. (4) and (10) cannot be written as an exact power with exponent $N$.

In Fig. 9 we report the Monte Carlo results for the inextensible WLC model: while the Helmholtz family of curves converges from the top to the bottom, upon increasing the polymer length, the Gibbs curves follow the opposite trend. We remark once more the convergence to a central common curve, representing the behavior of the system when the thermodynamic limit is reached. The example summarized in Fig. 9 corresponds to the value $\kappa=10 k_{B} T$ for the bending modulus at $T=293 \mathrm{~K}$. This value is comparable to that of polymer chains of biological interest (for example, for DNA $\kappa=15 k_{B} T$ (Refs. 15, 19, and 20)).

These results can be compared with the approximations published in literature for the WLC model. A first interpolation formula was given by the classical Marko and Siggia result $^{15}$

$$
\frac{f l}{k_{B} T}=\frac{l}{L_{p}}\left[\frac{1}{4(1-\zeta)^{2}}-\frac{1}{4}+\zeta\right]
$$

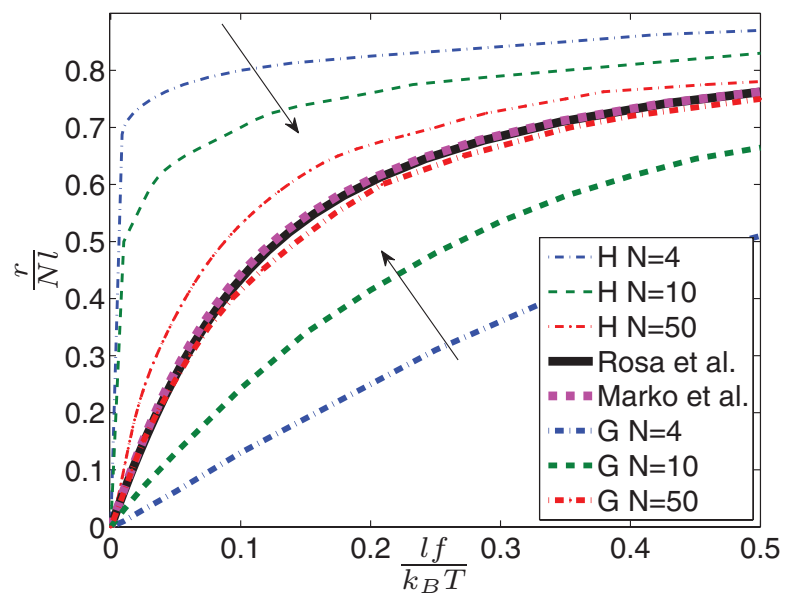

FIG. 10. Same as Fig. 9 where the approximated solutions by Marko and Siggia (see Eq. (41) taken from Ref. 15) and by Rosa et al. (see Eq. (42) taken from Refs. 19 and 20) are reported for the sake of comparison.

where $\zeta=r /(N l)$ is the polymer extension normalized to the contour length and $L_{p}=l_{\kappa} /\left(k_{B} T\right)$ is the persistence length. This result is asymptotically exact both in the large- and small-force limits of the continuous WLC model. Another, more recent result was derived for the discrete version of the WLC model, where the finite size of the equilibrium length $l$ is also accounted for ${ }^{19,20}$

$$
\begin{aligned}
\frac{f l}{k_{B} T}= & \frac{2 L_{p}}{l}\left[\sqrt{1+\left(\frac{l}{2 L_{p}}\right)^{2} \frac{1}{(1-\zeta)^{2}}}-\sqrt{1+\left(\frac{l}{2 L_{p}}\right)^{2}}\right] \\
+ & {\left[3 \frac{1-\mathcal{L}\left(\frac{L_{p}}{l}\right)}{1+\mathcal{L}\left(\frac{L_{p}}{l}\right)}-\frac{\frac{l}{2 L_{p}}}{\sqrt{1+\left(\frac{l}{2 L_{p}}\right)^{2}}}\right] \zeta }
\end{aligned}
$$

with $\mathcal{L}(x)=\operatorname{coth} x-1 / x$ being the Langevin function. It is easy to verify that in the continuum limit $l \rightarrow 0$ one recovers Eq. (41), as expected. Other interesting expressions for the force-extension curves can be found in Ref. 33. In Fig. 10 we report an enlarged detail of Fig. 9 together with the constitutive equations given in Eqs. (41) and (42): plots corresponding to both analytical approximations are contained between the Gibbs and Helmholtz Monte Carlo solutions for the larger value of $N$ considered. Therefore, the interpolation formulas in Eqs. (41) and (42) are in very good agreement with the behaviour of the WLC model when the thermodynamic limit is reached $(N>50$ in this case).

We can next analyse the convergence toward the thermodynamic limit. To this aim, we consider again the data in Fig. 9 and the ratio $\frac{r_{H}(N)}{r_{G}(N)}$ at a given fixed value of the normalized force. As before, it is found that Monte Carlo simulations are nicely fitted by the power law

$$
\frac{r_{H}(N)}{r_{G}(N)}=1+\frac{a}{N^{\alpha}},
$$

where $a$ and $\alpha$ are fitting parameters. All sets of data have been interpolated by linear regression in Fig. 11 and they provided the same scaling exponent $\alpha=1.30 \pm 0.05$. 


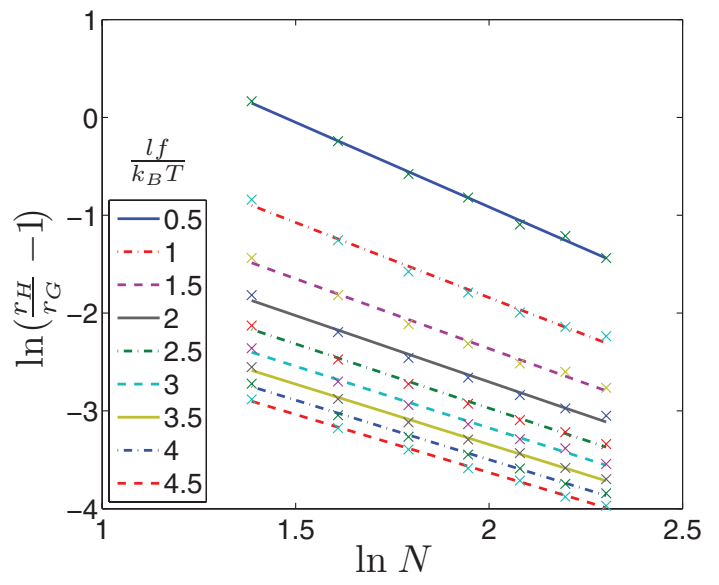

FIG. 11. Comparison between the elongations $r_{H}$ and $r_{G}$ for the pure WLC model. The elongations are calculated, respectively, in the Helmholtz and Gibbs ensembles as a function of the polymer length (given by the number $N$ of monomers in the chain). All sets of data are nicely fitted by Eq. (43) with the same scaling exponent $\alpha=1.30 \pm 0.05$.

We finally consider the semiflexible discrete WLC model with extensible bonds. We set the parameters $\kappa=10 k_{B} T$ (for the bending) and $k=10 k_{B} T /(\mathrm{nm})^{2}$ (for the stretching). In Fig. 12 we report the Monte Carlo results for both ensembles: the two families of curves are converging (from the top for the Helmholtz case and from the bottom for the Gibbs one) to the same constitutive relation for an increasing contour length, or number of monomers $N$.

As before, the curves in Fig. 12 can be used to analyse the convergence toward the thermodynamic limit through Eq. (43). Figure 13 shows that in this case the linear regression leads to a scaling exponent $\alpha=1.40 \pm 0.05$ for any value of the normalized force.

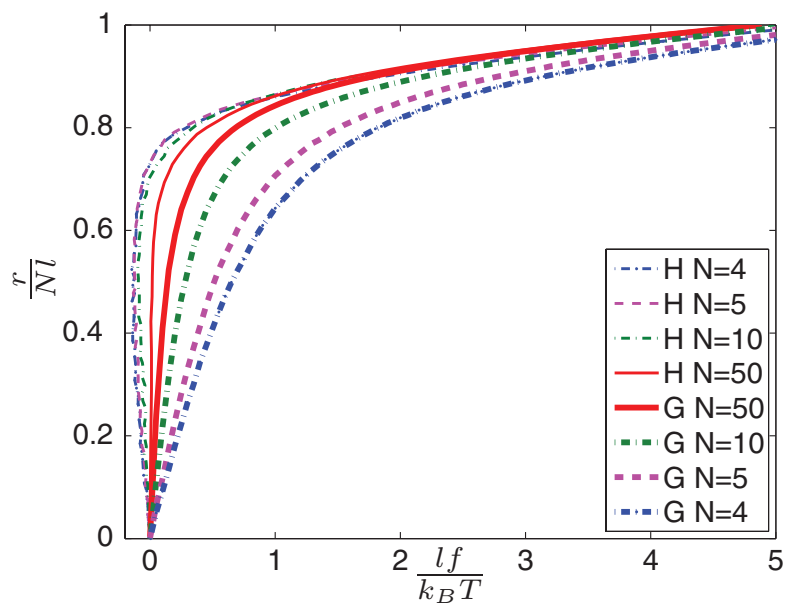

FIG. 12. Constitutive relation provided by Monte Carlo simulations for the WLC model with elastic bonds between the monomers, both under Helmholtz $(H)$ and Gibbs $(G)$ boundary conditions. The elongation $r$ and the traction $f$ are reported, respectively, on the vertical and horizontal axis in dimensionless units (see text). The Helmholtz constitutive relation is reported for different polymer lengths, given by the number $N$ of monomers in the chain.

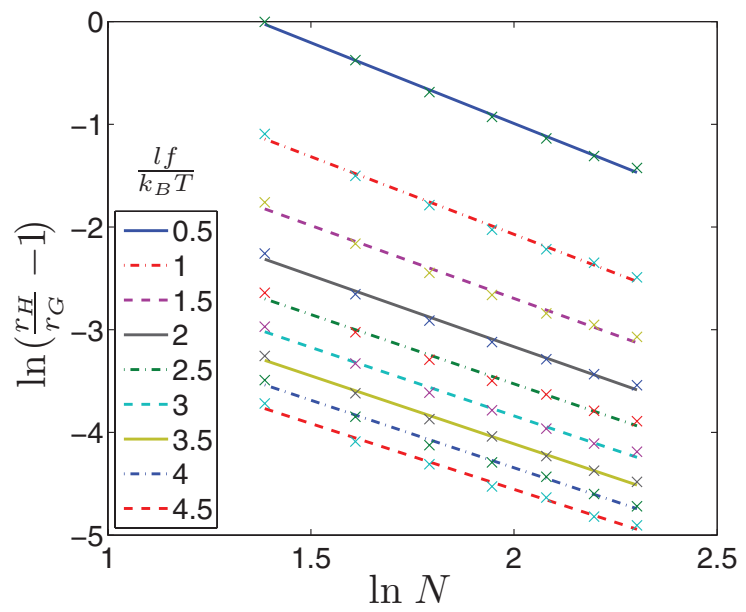

FIG. 13. Comparison between the elongations $r_{H}$ and $r_{G}$ for the WLC model with elastic bonds between the monomers. The elongations are calculated, respectively, in the Helmholtz and Gibbs ensembles as a function of the polymer length (given by the number $N$ of monomers in the chain). All sets of data are nicely fitted by Eq. (43) with the same scaling exponent $\alpha=1.40 \pm 0.05$.

\section{CONCLUSIONS}

In this work we investigated how the force-extension curve of a model polymer chain is affected by the loading protocol which can typically be fixed-ends or fixed-force. We showed how such macroscopic boundary conditions can be formulated within the Helmholtz and the Gibbs ensembles of the statistical mechanics. We adopted flexible and semiflexible polymer models, with and without extensible bonds. Extensible bonds were here described by linear springs for simply comparing analytical and Monte Carlo results. However, the present approach can be easily extended to more complex, nonlinear springs. ${ }^{47}$

After a theoretical introduction on different boundary conditions, we discussed the notion of thermodynamic limit. In particular, we proved that different experimental strategies used for stretching the polymer lead to the same results when the number of monomers is large enough. On the other hand, by Monte Carlo simulations we showed that for short chains the two ensembles are characterized by very different elastic behaviors. In all cases here investigated (FJC, WLC, and their extensible versions) we found that the convergence to the thermodynamic limit is well described by suitable power laws with well defined scaling exponents. More specifically, we proved that such power laws can fit the Monte Carlo results with high accuracy and that different polymer models have different scaling exponents.

The results of this work and the demonstration of different scaling laws may help to discriminate the response of polymers with different internal chemical structure, in the short-length limit. A similar treatment can be followed also for different deformation modes. For example, the twisting experiments can be performed by means of magnetic tweezers: ${ }^{48}$ the equivalent of the present fixed-ends versus fixed-force scheme would be substituted with the fixed-twist versus fixed-torque scheme. 


\section{ACKNOWLEDGMENTS}

We acknowledge computational support by CASPUR (Rome, Italy) under project "Standard HPC Grant 2010/2011." F.M. acknowledges the Department of Physics of the University of Cagliari for the extended visiting grant and the Cnrs-IEMN Lille for the kind hospitality offered during part of this work. L.C. acknowledges financial support by Regional Government of Sardinia under the project "Ricerca di Base" titled "Modellizzazione Multiscala della Meccanica dei Materiali Complessi" (RAS-M4C). R.Z. thanks a postdoc grant from IEMN Cnrs, Axe-1 "Nanostructures," and a supporting grant from the CPER Region Nord-Pas de Calais. Discussions with R. Blossey (Interdisciplinary Research Institute, Lille) are also acknowledged.

${ }^{1}$ F. Ritort, J. Phys.: Condens. Matter 18, R531 (2006).

${ }^{2}$ K. R. Chaurasiya, T. Paramanathan, M. J. McCauley, and M. C. Williams, Phys. Life Rev. 7, 299 (2010).

${ }^{3}$ A. Ashkin, Proc. Natl Acad. Sci. U.S.A. 94, 4853 (1997).

${ }^{4}$ C. Gosse and V. Croquette, Biophys. J. 82, 3314 (2002).

${ }^{5}$ D. M. Czajkowsky and Z. Shao, FEBS Lett. 430, 51 (1998).

${ }^{6}$ C. R. Calladine, H. R. Drew, B. F. Luisi, and A. A. Travers, Understanding DNA: The Molecule and How it Works (Elsevier/Academic, Amsterdam, 1992).

${ }^{7}$ H. Yang, G. Luo, P. Karnchanaphanurach, T. M. Louie, I. Rech, S. Cova, L. Xun, and X. S. Xie, Science 302, 262 (2003).

${ }^{8}$ E. A. Lipman, B. Schuler, O. Bakajin, and W. A. Eaton, Science 301, 1233 (2003)

${ }^{9}$ F. Jülicher, A. Adjari, and J. Prost, Rev. Mod. Phys. 69, 1269 (1997).

${ }^{10}$ M. Rief, F. Oesterhelt, B. Heymann, and H. E. Gaub, Science 275, 28 (1997).

${ }^{11}$ J. Liphardt, S. Dumont, S. B. Smith, I. Tinoco, and C. Bustamante, Science 296, 1832 (2002).

${ }^{12}$ F. Cleri, Sci. Model. Simul. 15, 369 (2008).

${ }^{13}$ A. Alemany and F. Ritort, Europhys. News 41, 27 (2010).

${ }^{14}$ S. B. Smith, L. Finzi, and C. Bustamante, Science 258, 1122 (1992).

${ }^{15}$ J. F. Marko and E. D. Siggia, Macromolecules 28, 8759 (1995).

${ }^{16}$ J. M. Huguet, C. V. Bizarro, N. Forns, S. B. Smith, C. Bustamante, and F. Ritort, Proc. Natl. Acad. Sci. U.S.A. 107, 15341 (2010).

${ }^{17}$ R. D. Kamien, Rev. Mod. Phys. 74, 953 (2002).

${ }^{18}$ C. Anselmi, P. DeSantis, and A. Scipioni, Biophys. Chem. 113, 209 (2005).
${ }^{19}$ A. Rosa, T. X. Hoang, D. Marenduzzo, and A. Maritan, Macromolecules 36, 10095 (2003).

${ }^{20}$ A. Rosa, T. X. Hoang, D. Marenduzzo, and A. Maritan, Biophys. Chem. 115, 251 (2005).

${ }^{21}$ P. K. Purohit, M. E. Arsenault, Y. Goldmanb, and H. H. Bau, Int. J. NonLinear Mech. 43, 1056 (2008).

${ }^{22}$ T. Su and P. K. Purohit, J. Mech. Phys. Solids 58, 164 (2010).

${ }^{23}$ J. H. Weiner and M. R. Pear, Macromolecules 10, 317 (1977).

${ }^{24}$ R. M. Neumann, Phys. Rev. A 31, 3516 (1985).

${ }^{25}$ D. Keller, D. Swigon, and C. Bustamante, Biophys. J. 84, 733 (2003).

${ }^{26}$ R. M. Neumann, Biophys. J. 85, 3418 (2003).

${ }^{27}$ T. Strick, J.-F. Allemand, V. Croquette, and D. Bensimon, Prog. Biophys. Mol. Biol. 74, 115 (2000).

${ }^{28}$ M. D. Wang, H. Yin, R. Landick, J. Gelles, and S. M. Block, Biophys. J. 72, 1335 (1997).

${ }^{29}$ P. Cluzel, A. Lebrun, R. Lavery, J.-L. Viovy, D. Chatenay, and F. Caron, Science 271, 792 (1997).

${ }^{30}$ T. Hugel, M. Grosholz, H. Clausen-Schaumann, A. Pfau, H. Gaub, and M. Seitz, Macromolecules 34, 1039 (2001).

${ }^{31}$ X. Liu and G. H. Pollack, Biophys. J. 83, 2705 (2002).

${ }^{32}$ C. Bouchiat, M. D. Wang, J.-F. Allemand, T. Strick, M. Block, and V. Croquette, Biophys. J. 76, 409 (1999).

${ }^{33}$ J. Kierfeld, O. Niamploy, V. Sa-yakanit, and R. Lipowsky, Eur. Phys. J. E 14, 17 (2004).

${ }^{34}$ J. W. Gibbs, Elementary Principles in Statistical Mechanics (Charles Scribner's Sons, New York, 1902).

${ }^{35}$ J. H. Weiner, Statistical Mechanics of Elasticity (Dover, New York, 2002).

${ }^{36}$ A. Erdelyi, Asymptotic Expansions (Dover, New York, 1956).

${ }^{37}$ R. R. Netz, Macromolecules 34, 7522 (2001).

${ }^{38}$ M. Abramowitz and I. A. Stegun, Handbook of Mathematical Functions (Dover, New York, 1970).

${ }^{39}$ M. Rubinstein and R. H. Colby, Polymer Physics (Oxford University Press, New York, 2003).

${ }^{40}$ H. J. Kreuzer and S. H. Payne, Phys. Rev. E 63, 021906 (2001).

${ }^{41}$ S. Sinha and J. Samuel, Phys. Rev. E 71, 021104 (2005).

${ }^{42}$ A. Dhar and D. Chaudhuri, Phys. Rev. Lett. 89, 065502 (2002).

${ }^{43}$ K. Binder, Rep. Progr. Phys. 60, 487 (1997).

${ }^{44}$ D. Frenkel and B. Smit, Understanding Molecular Simulation (Academic, San Diego, 1996).

${ }^{45}$ M. P. Allen and D. J. Tildesley, Computer Simulations of Liquids (Clarendon, Oxford, 1987).

${ }^{46}$ M. A. Miller, L. M. Amon, and W. P. Reinhardt, Chem. Phys. Lett. 331, 278 (2000).

${ }^{47}$ J. R. Blundell and E. M. Terentjev, Soft Matter 7, 3967 (2011).

${ }^{48}$ M. Tanase, N. Biais, and M. Sheetz, Methods Cell Biol. 83, 473 (2007). 